\title{
ES Cell Line
}

National Cancer Institute

\section{Source}

National Cancer Institute. ES Cell Line. NCI Thesaurus. Code C20232.

Stable cell lines derived from embryonic stem cells. They can be propagated indefinitely in the primitive undifferentiated state while remaining pluripotent. 